Keywords: HPV self-sampling; non-attenders; cervical cancer screening; HPV primary screening; cervical intraepithelial neoplasia; implementation; Denmark

\title{
High-grade cervical intraepithelial neoplasia in human papillomavirus self-sampling of screening non-attenders
}

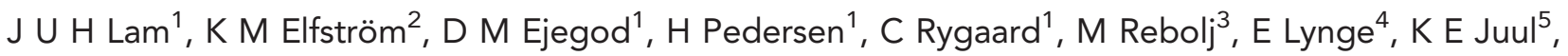 \\ $\mathrm{S} K \mathrm{Kj}^{5,6}$, J Dillner ${ }^{2,7}$ and J Bonde*,1,3 \\ ${ }^{1}$ Department of Pathology, Copenhagen University Hospital Hvidovre, Kettegård Allé 30, 2650 Hvidovre, Copenhagen, Denmark; \\ ${ }^{2}$ Department of Laboratory Medicine, Karolinska Institutet, Stockholm 14186, Sweden; ${ }^{3}$ Clinical Research Centre, Copenhagen \\ University Hospital Hvidovre, Kettegård Allé 30, 2650 Hvidovre, Copenhagen, Denmark; ${ }^{4}$ Department of Public Health, University \\ of Copenhagen, Copenhagen 1014, Denmark; ${ }^{5}$ Unit of Virus, Lifestyle and Genes, Danish Cancer Society Research Center, \\ Copenhagen 2100, Denmark; ${ }^{6}$ Department of Obstetrics and Gynecology, Copenhagen University Hospital Rigshospitalet, \\ Copenhagen 2100, Denmark and ${ }^{7}$ Department of Medical Epidemiology and Biostatistics, Karolinska Institutet, Stockholm 14186, \\ Sweden
}

Background: Self-sampling for human papillomavirus (HPV) offered to women who do not participate in cervical cancer screening is an increasingly popular method to increase screening coverage. The rationale behind self-sampling is that unscreened women harbour a high proportion of undetected precancer lesions. Here, we compare the cervical intraepithelial neoplasia grade 2 or worse ( $\geqslant C I N 2)$ detection rate between non-attenders who participated in self-sampling and women attending routine screening.

Methods: A total of 23632 women who were qualified as non-attenders in the Copenhagen Region were invited for HPV-based self-sampling. Of these, 4824 women returned a self-sample, and HPV-positive women were referred for cytology and HPV cotesting as follow-up. The entire cohort and a reference cohort (3347 routinely screened women) were followed for histopathology confirmed $\geqslant C I N 2$. Odds ratio $(O R)$ and the relative positive predictive value of $\geqslant C I N 2$ detection between the two populations were estimated.

Results: Women participating in self-sampling had a higher $\geqslant C I N 2$ detection than women undergoing routine cytology-based screening (OR $=1.83,95 \% \mathrm{Cl}: 1.21-2.77)$ and a similar detection as routinely screened women tested with cytology and HPV testing ( $\mathrm{OR}=1.03,95 \% \mathrm{Cl}$ : $0.75-1.40)$. The positive predictive value for $\geqslant \mathrm{CIN} 2$ was higher in screening non-attenders than in routinely HPV- and cytology-screened screened women (36.5\% vs $25.6 \%$, respectively).

Conclusions: Self-sampling offered to non-attenders showed higher detection rates for $\geqslant C I N 2$ than routine cytology-based screening, and similar detection rates as HPV and cytology co-testing. This reinforces the importance of self-sampling for screening non-attenders in organised cervical cancer screening.

A major challenge in preventing cervical cancer is the large proportion of women who are screening non-attenders. In the organised Danish cervical cancer screening programme, women aged 23-49 years are invited every 3 years for screening, and women 50-65 years of age are invited every 5 years. Approximately $75 \%$ of the target population is screened following these recommendations, with minor regional and annual fluctuations (Danish Quality Assurance Database for the Cervical Cancer

*Correspondence: Dr J Bonde; E-mail: jesper.hansen.bonde@regionh.dk 
Screening Program). Yet, $45 \%$ of new cancer cases are diagnosed among non-attenders (Dugue et al, 2012).

Human papillomavirus (HPV)-based self-sampling has been shown to increase screening participation in several studies (Gok et al, 2010; Enerly et al, 2016; Sultana et al, 2016). In May 2014, we initiated 'The Copenhagen Self-sampling Initiative' (CSi) pilot to gain experience in how to best offer non-attenders in the Capital Region of Denmark an HPV self-sampling test as an alternative to the standard physician-taken cytology sample. The CSi was designed as an implementation opt-in pilot study, where approximately half of the women residing in the Capital Region who had missed at minimum one screening round were invited to order a self-sampling brush. As the pilot implementation was population based, the invited women had variable screening history, ranging from those last screened 4 years before the self-sampling invitation to those who had never been screened. Overall, $\sim 20 \%$ of the 23632 invited women participated by returning a self-sampling brush. Of these, $40 \%$ were long-term unscreened with no registered cytology in $\geqslant 10$ years (Lam et al, 2017). By November 2016, an additional 18\% of the invited women passively or actively declined the selfsampling invitation and instead went to their own general practitioner (GP) for screening. In total, 38\% of all invited women were screened in an 18-month period after receiving the self-sampling invitation.

A successful self-sampling strategy should not be less sensitive for cervical intraepithelial neoplasia grade 2 or worse ( $\geqslant$ CIN2) detection than routine screening. We investigated the detection rate of $\geqslant \mathrm{CIN} 2$ among the screened women in a real-life selfsampling setting, the $\mathrm{CSi}$, and estimated the positive predictive value of this procedure (with $\geqslant \mathrm{CIN} 2$ detection as a threshold). Furthermore, we compared the observations to a population-based routine screening cohort from the Horizon study (Preisler et al, 2013, 2016; Rebolj et al, 2013, 2014a, b, 2015a, 2016a, b; Bonde et al, 2014; Ejegod et al, 2015). Samples from both cohorts were tested in the same laboratory.

\section{MATERIALS AND METHODS}

The 23632 screening non-attenders in the CSi implementation study were invited to participate in screening via self-sampling using an Evalyn brush (Rovers, Oss, The Netherlands). Nonattenders were defined as women who had not been screened for at least 4 (if aged 27-49 years) or 6 years (if aged 50-65 years). Invitations for self-sampling were sent in batches of 1000 women from May 2014 to April 2015; during this time, new routine screening invitations may have been sent from the screening programme. The women invited for self-sampling were sent a letter with information on cervical cancer screening and the association between HPV and cervical cancer; they could order a self-sampling brush from our laboratory and return it using a prestamped envelope. In total, 4865 returned their self-sampling test for HPV testing on two or three different HPV assays (Figure 1). An additional 4291 women were not screened via self-sampling, but screened by a GP after receiving the self-sampling invitation letter. Note that these numbers differ from those reported previously (Lam et al, 2017), owing to a longer follow-up (November 2016 instead of December 2015). The HPV positivity was determined on the CLART (Genomica, Madrid, Spain) and Onclarity (BD, Sparks, MD, USA) assays for all samples. A subset of the first 1008 samples was also analysed on Hybrid Capture 2 (HC2; Qiagen, Hilden, Germany). Upon a positive HPV self-sample (i.e., if any of the three HPV assays gave a positive test result), women were referred to a GP-taken cytology follow-up sample. The follow-up samples were co-tested with HPV and cytology. Based upon the outcome of this follow-up test result, women were referred to re-testing or gynaecology examinations. For the current study, the women included in CSi were followed for 18 months (until November 2016) after the last invitations were sent.

For comparison, we have used data from the Horizon study that was a population-based study of women routinely screened at their GP. It included residual material from 5034 consecutive SurePath cytology samples from women aged 16-89 years. The samples were additionally tested for HPV using four different HPV assays (HC2, cobas, CLART, and APTIMA), as described previously (Preisler et al, 2013, 2016; Rebolj et al, 2013, 2014a, b, 2015a, 2016a, b; Bonde et al, 2014; Ejegod et al, 2015). Horizon was nested into routine practice. The women were therefore triaged according to routine practice if they had cytology abnormal findings (defined as atypical squamous cells of undetermined significance, $\geqslant$ ASCUS). As an extra intervention in the Horizon study, cytology normal and HPV-positive women had an active follow-up according to the study protocol (Figure 2), where these women were invited for repeat testing after 18 months.

Both studies were conducted at the Department of Pathology, Copenhagen University Hospital (Supplementary Table 1). The department was responsible for sending screening invitations and testing of all cervical samples in the Copenhagen and Frederiksberg municipalities when the Horizon study was undertaken between June and August 2011 (this area covered $\sim 15 \%$ of all Danish women eligible for cervical screening). From 2012 onwards, the department was responsible for sending invitations and testing all samples from the whole Capital Region ( $\sim 33 \%$ of all eligible women), where the CSi was implemented between May 2014 and November 2015.

In order to ensure comparability of the cohorts, we excluded women from the Horizon study who were outside the selfsampling age range of $27-65$ years $(n=1040)$, as well as women with referral samples (which was women who had ASCUS or more severe abnormality diagnosis registered in the previous 15 months, or were HPV positive in the past 12 months, $n=647)$. Finally, the two populations, Horizon $(n=3347)$ and CSi $(n=23632)$, were included in the analysis.

Data sources. Women's screening status and history were retrieved from the National Pathology Database (Patobank). Patobank is a nationwide registry that covers all specimens from all pathology departments in Denmark, and has been highly complete since mid-2000s (Bjerregaard and Larsen, 2011). Women's screening status was determined by calculating the time between the most recent test on record between 1 January 2000 and 6 May 2014, when we retrieved the list of eligible women to be invited for self-sampling. For the Horizon study, this was between 1 January 2000 and the date the Horizon study sample was collected in the routine laboratory.

If the referral, GP-taken, follow-up sample was cytology normal and HPV positive, women were recommended for retesting after 12 months. The data for the CSi study were retrieved 18 months after the last invitations were sent out, in November 2016. In the Horizon study, cytology-normal and HPV-positive women were invited by the laboratory to be retested after 18 months. The histology follow-up took place until December 2013 that was $\sim 30$ months after study start.

Statistical analysis. Means and proportions were used to describe the study populations. The outcome of interest was detection of CIN2 or worse $(\geqslant \mathrm{CIN} 2)$ vs normal biopsy/CIN1/no biopsy. All abnormal histological findings in the CSi study were confirmed by a chief physician reviewing the description, diagnosis code, and electronic medical records. Odds ratios (ORs), their 95\% confidence intervals (CIs), and $P$-values of the association between $\geqslant \mathrm{CIN} 2$ detection and explanatory variables were estimated in a logistic regression model. The ORs were reported as crude and 


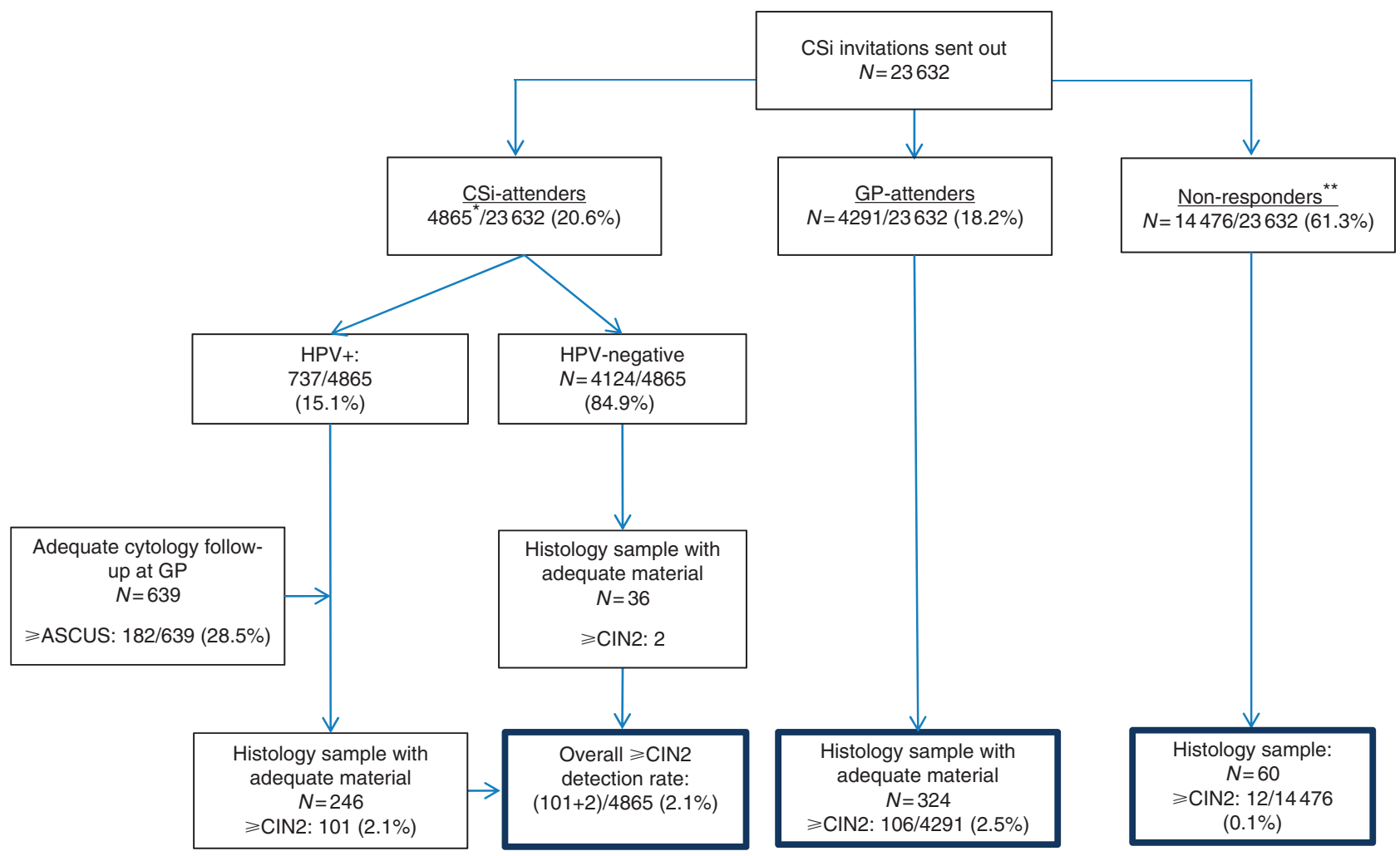

Figure 1. Flowchart of the CSi study design, follow-up triage, and $\geqslant \mathrm{CIN} 2$ detection rate. ${ }^{*}$ Of all tested samples, 4 were invalid on all HPV assays, 773 samples had a positive test result on any of the three assays, and 246 of these had biopsies taken. Of the 4124 HPV-negative women, 36 had a biopsy taken (for an unknown reason). Because of a hardware failure on the Onclarity assay, some women received their result based on CLART and HC2 only. The samples were later re-tested on Onclarity, with 36 women having a positive test result. Owing to hospital practice, these women did not receive a supplement result. The flowchart illustrates the follow-up process based on the result the women received, that is, the result that influenced their clinical management. ${ }^{\star \star}$ Non-responders remained unscreened during the whole study period; the CIN2 or worse cases detected might be symptom-related indications rather than screening. $\mathrm{CIN}=$ cervical intraepithelial neoplasia.

adjusted, where age, study and the women's screening history were taken into account. Cofactors were examined separately and as potential confounders. Age and screening history were determined to be a confounder of the association between the study population, the exposure of interest, and $\geqslant \mathrm{CIN} 2$ detection, and were therefore included in the adjusted model.

Four mutually exclusive population types were defined for the analysis: one for the participants in the Horizon study, and three for the women included in the CSi study: CSi-attenders (women who returned their self-sampling test), GP-attenders (women who had a physician-taken cytology sample), and non-responders (invited women who neither responded to the CSi invitation nor were screened by a GP).

Positive predictive value of $\geqslant \mathrm{CIN} 2$ detection was calculated as the proportion of $\geqslant \mathrm{CIN} 2$ cases found among those who had an adequate biopsy taken.

Screening history was categorised into two groups: intermittently screened in case the women's latest screening samples were registered within 10 years before the study, and long-term unscreened in case where the women's last screening samples, if any, were registered $\geqslant 10$ years before the study.

All statistical analyses were performed using Stata 13.1. (StataCorp, College Station, TX, USA).

Ethical approvals. Linkage of the data for both CSi and Horizon was approved by the Danish Data Protection Agency under notification number AHH-2015-084 I-Suite number: 04139, and AHH-2015-084, I-Suite number: 04139, respectively. HPV testing in the Horizon study did not require ethical approval, as it was undertaken as a quality development study in concordance with the Committee Act under The National Committee on Health Research Ethics (2011 §14, part 3); the 18-month follow-up round was approved by the Ethics Committee of the Danish Capital Region (journal no. H-4-2012-120), and the women provided informed consent. The CSi was a pilot implementation, mandated by the Danish Health Authority and the Capital Region of Denmark; ethical approval was therefore not required. Women who ordered the self-sampling kit implicitly agreed to participate in the implementation.

\section{RESULTS}

Of the 23632 women invited to participate in self-sampling, 4865 (20.6\%) returned a self-sampling test, typically within the first 6 months, $4291(18.2 \%)$ had a cytology sample taken by a GP, and $14476(61.3 \%)$ had neither within the register-based follow-up period (Figure 1). Of the $4865 \mathrm{CSi}$-attenders (women who returned a self-sampling test), 737 (15.1\%) were HPV positive, and referred for an immediate routine cytology sample with HPV co-testing. In total, 639 (86.8\%) women had cytology and HPV follow-up, among whom $98 \geqslant \mathrm{CIN} 2$ were detected. Six HPV-positive women instead opted for a direct colposcopy, and three of these had $\geqslant$ CIN2. Two additional $\geqslant$ CIN2 were detected among HPVnegative CSI-attenders, despite not being recommended for additional testing. In total, therefore, $103(2.1 \%) \geqslant \mathrm{CIN} 2$ were detected among $4865 \mathrm{CSi}$-attenders (Table 1). Among the 4291 GP-attenders, $106(2.5 \%)$ had a diagnosis of $\geqslant C I N 2$, whereas 12 out of $14476(0.1 \%)$ women from the non-responders group had a $\geqslant$ CIN2 detected; the reason for these biopsies is unknown, but 


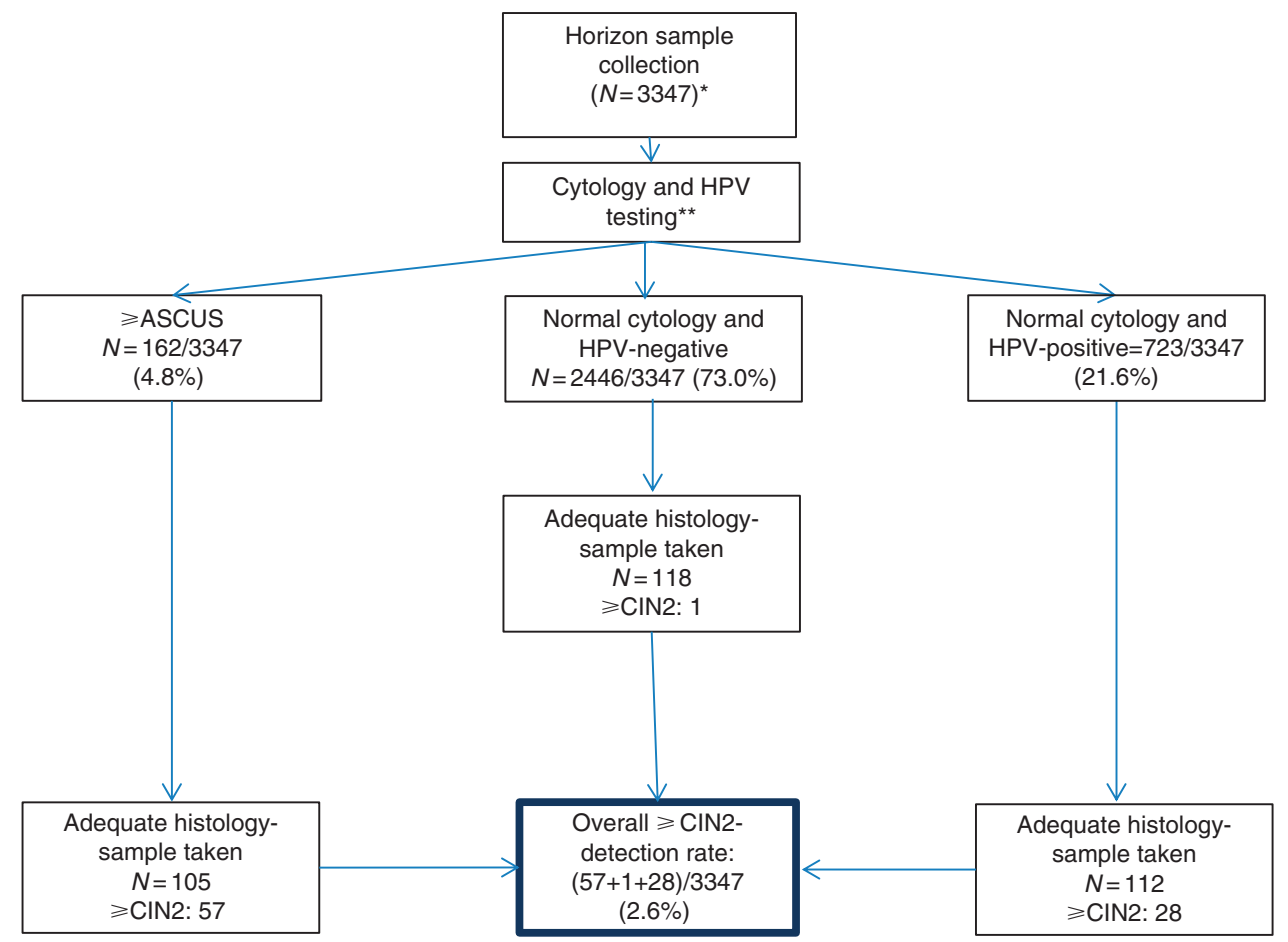

Figure 2. Flowchart of the Horizon study design, follow-up triage, and $\geqslant \mathrm{CIN} 2$ detection. * Of the 3347 screened women, 16 (0.48\%) were inadequate for cytology reading. One of the women had a histology sample registered with normal findings. This contributed to a total of 336 biopsies taken. ${ }^{\star}$ Women were followed-up according to routine practice based on the cytology result only. An active follow-up was performed on women with cytology normal HPV-positive findings according to the study protocol that was repeat testing after 18 months. Women with normal cytology and negative HPV test results were referred back to the routine screening programme; some of these had a histology sample taken despite the recommendations. Women who were cytology negative and HPV positive were invited for repeated testing after 18 months. All HPV test results reported here took into account the testing results on any of the four assays. $\mathrm{CIN}=$ cervical intraepithelial neoplasia.

may have been taken in response to symptoms - six of these lesions were cervical cancer (Table 1).

In the Horizon study (Figure 2), 3347 screened women were offered follow-up in case they had abnormal cytology or a positive HPV test result on any of the four assays. The remaining women were referred back to the routine screening programme. Overall, 336 histology diagnoses were retrieved with $86 \geqslant \mathrm{CIN} 2(2.6 \%)$ in the Horizon study. When excluding the $\geqslant$ CIN2 found among women in the per-protocol arm (repeat testing for women cytology normal and HPV positive), and only including the $\geqslant$ CIN2 found in the cytology stand-alone protocol (which reflects the routine screening programme), the $\geqslant$ CIN2 detection was 58/3347 (1.7\%).

The CSi-attenders were on average 7 years older than GPattenders in CSi and in the Horizon study (mean ages: 47.1 vs 40.3 vs 40.6 years, respectively). Almost all (94.2\%) women in the Horizon study were screened at least once in the preceding 10 years. This was the case for approximately two-thirds of CSiattenders $(60.1 \%)$ and GP-attenders $(69.2 \%)$, but for only $36.0 \%$ of the CSi non-responders (who were also non-responders to routine screening). Among the adequate biopsies, CSi-attenders and GPattenders had a higher detection of $\geqslant \mathrm{CIN} 2$ (positive predictive value of an adequate biopsy: $36.5 \%$ and $32.7 \%$, respectively) than non-responders $(20.0 \%)$ and women included in the Horizon study (25.6\%). Among all detected $\geqslant \mathrm{CIN} 2$, women were slightly more likely to have $\geqslant \mathrm{CIN} 3$ detected if they were CSI-attenders $(78.6 \%$ of all $\geqslant \mathrm{CIN} 2$ diagnoses were $\geqslant \mathrm{CIN} 3$ ) or GP-attenders $(78.3 \%)$ than when they were routinely screened (72.1\%, Horizon). In total, 18 women were diagnosed with cervical cancer in the CSi study, and one in the Horizon study.

Women aged $>30$ years were significantly less likely to have a $\geqslant$ CIN2 diagnosis $(P<0.01)$ compared with women aged 27-29 years, and this did not change after adjustment for population type and screening history (Table 2). Without any adjustment, intermittently screened women (women who were screened within the past 10 years) appeared to have a higher risk of $a \geqslant \mathrm{CIN} 2$ diagnosis than long-term unscreened women $(P<0.01)$. After adjustment for age and population type, no significant difference was observed between intermittently and long-term unscreened women $(P=0.97)$. CSi-attenders $(P=0.18)$ and GP-attenders $(P=0.78)$ appeared to have a similar (crude) risk of $\geqslant \mathrm{CIN} 2$ detection as women screened in the Horizon study that remained virtually unchanged after adjustment for age and screening history ( $P=0.88$ and $P=0.81$, respectively). This was also the case if screening history was re-defined as either the number of unscreened years or dichotomised as unscreened for $<6$ and $\geqslant 6$ years ( $P=0.40$ and $P=0.78$, respectively; data not reported). When we excluded the effect of HPV-based screening in the Horizon study and compared the $\geqslant \mathrm{CIN} 2$ detection in CSi only with cytology-based detection in Horizon (i.e., routine cytologybased screening), CSi-attenders had a 1.83 times higher detection of $\geqslant \mathrm{CIN} 2$ (adjusted $\mathrm{OR}=1.83,95 \% \mathrm{CI}: 1.21-2.77, P<0.01$ ). The CSi non-responders had a significantly lower detection of $\geqslant$ CIN2 than routinely screened women $(P<0.01)$.

\section{DISCUSSION}

In our population-based study of screening non-attenders, HPVbased self-sampling detected a similar proportion of $\geqslant \mathrm{CIN} 2$ as routine screening with cytology and HPV-based co-testing, and a higher proportion than stand-alone routine cytology-based screening. Furthermore, a slightly higher proportion of CIN3 and cervical cancer was also observed among the CSi-attenders. The positive predictive value for $\geqslant \mathrm{CIN} 2$ of a biopsy was slightly higher in CSi 
Table 1. The characteristics of the two study populations

\begin{tabular}{|c|c|c|c|c|c|c|c|c|}
\hline \multirow{2}{*}{ Mean age (years; 95\% Cl) } & \multicolumn{6}{|c|}{$\operatorname{CSi}(N=23632)$} & \multicolumn{2}{|c|}{ Horizon $(N=3347)$} \\
\hline & \multicolumn{2}{|c|}{$\begin{array}{l}\text { CSi-attenders } \\
(n=4865)\end{array}$} & \multicolumn{2}{|c|}{$\begin{array}{l}\text { GP-attenders } \\
(n=4291)\end{array}$} & \multicolumn{2}{|c|}{$\begin{array}{l}\text { Non-responders } \\
(n=14476)\end{array}$} & \multicolumn{2}{|c|}{$\begin{array}{l}\text { GP-taken sample } \\
\quad(n=3347)\end{array}$} \\
\hline $\begin{array}{l}\text { Age group (years) } \\
27-29 \\
30-39 \\
40-49 \\
50-59 \\
60-65 \\
\text { Total }\end{array}$ & $\begin{array}{r}385 \\
1092 \\
1215 \\
1275 \\
898 \\
4865\end{array}$ & $\begin{array}{r}7.9 \% \\
22.4 \% \\
25.0 \% \\
26.2 \% \\
18.5 \% \\
100.0 \%\end{array}$ & $\begin{array}{r}588 \\
1657 \\
1311 \\
514 \\
221 \\
4291\end{array}$ & $\begin{array}{r}13.7 \% \\
38.6 \% \\
30.6 \% \\
12.0 \% \\
5.1 \% \\
100.0 \%\end{array}$ & $\begin{array}{r}1318 \\
2962 \\
3107 \\
4099 \\
2990 \\
14476\end{array}$ & $\begin{array}{r}9.1 \% \\
20.5 \% \\
21.5 \% \\
28.3 \% \\
20.7 \% \\
100.0 \%\end{array}$ & $\begin{array}{r}478 \\
1295 \\
897 \\
462 \\
215 \\
3347\end{array}$ & $\begin{array}{r}14.3 \% \\
38.7 \% \\
26.8 \% \\
13.8 \% \\
6.4 \% \\
100.0 \%\end{array}$ \\
\hline $\begin{array}{l}\text { Screening history } \\
\text { Long-term unscreened } \\
\text { Intermittently screened } \\
\text { Total }\end{array}$ & $\begin{array}{l}1621 \\
2440 \\
4061\end{array}$ & $\begin{array}{r}39.9 \% \\
60.1 \% \\
100.0 \%\end{array}$ & $\begin{array}{r}927 \\
2083 \\
3010\end{array}$ & $\begin{array}{r}30.8 \% \\
69.2 \% \\
100.0 \%\end{array}$ & $\begin{array}{r}7540 \\
4243 \\
11783\end{array}$ & $\begin{array}{r}64.0 \% \\
36.0 \% \\
100.0 \%\end{array}$ & $\begin{array}{r}133 \\
2158 \\
2291\end{array}$ & $\begin{array}{r}5.8 \% \\
94.2 \% \\
100.0 \%\end{array}$ \\
\hline $\begin{array}{l}\geqslant \text { CIN2 detection (regardless of the HPV test result) } \\
<\text { CIN2 } \\
\geqslant \text { CIN2 (positive predictive value) } \\
\text { Total }\end{array}$ & $\begin{array}{l}179 \\
103 \\
282\end{array}$ & $\begin{array}{r}63.5 \% \\
36.5 \% \\
100.0 \%\end{array}$ & $\begin{array}{l}218 \\
106 \\
324\end{array}$ & $\begin{array}{r}67.3 \% \\
32.7 \% \\
100.0 \%\end{array}$ & $\begin{array}{l}48 \\
12 \\
60\end{array}$ & $\begin{array}{r}80.0 \% \\
20.0 \% \\
100.0 \%\end{array}$ & $\begin{array}{r}250 \\
86 \\
336\end{array}$ & $\begin{array}{r}74.4 \% \\
25.6 \% \\
100.0 \%\end{array}$ \\
\hline $\begin{array}{l}\text { Positive predictive value of } \geqslant \mathrm{CIN} 2 \text { detection } \\
\text { compared with Horizon }\end{array}$ & & 0.01 & & 0.02 & & 0.93 & & rence \\
\hline
\end{tabular}

compared with Horizon. This indicated that self-sampling was effective and efficient in detecting high-grade CIN lesions whose treatment might prevent future cases of cervical cancer.

From an implementation point of view, the CSi and the Horizon studies illustrate the true impact of screening activities in a realworld setup. The $\geqslant \mathrm{CIN} 2$ rate found in the CSi therefore demonstrated the expected rates that would be found if selfsampling would be added as an additional screening option for women not attending routine screening.

Women who remained unscreened even after a self-sampling invitation were highly unlikely to have a $\geqslant \mathrm{CIN} 2$ lesion detected quite simply as they were not examined. Most of their CIN2 or CIN3 lesions would go undetected until overtly symptomatic. In our implementation study, $10 \%$ of all $\geqslant \mathrm{CIN} 2$ diagnoses in these women were cancer, compared with $\leqslant 2 \%$ in women undergoing routine or self-sampling screening. These women remain a research priority to determine a better way of attracting them to screening, although in younger birth cohorts this need will be attenuated owing to HPV vaccination.

Few studies have compared the $\geqslant \mathrm{CIN} 2$ detection in screening non-attenders who participate in self-sampling and in routinely screened women. Bais et al (2007) compared 736 women undergoing self-sampling (corresponding to our 'CSi-attenders' group) and an age-matched population-based cohort of 6208 routinely screened women (corresponding to the 'Horizon' population) as the reference group. This study found an overall higher $\geqslant$ CIN2 detection in the self-sampling group compared with routinely screened women $(\mathrm{OR}=2.59,95 \% \mathrm{CI}$ : $1.31-5.12)$. A similarly higher detection of $\geqslant \mathrm{CIN} 2$ was found for selfsampling participants compared with routine screening participants by Gok et al (2012), with a relative risk of 1.63 (95\% CI: 1.4-1.9). Sultana et al (2016), on the other hand, reported in an opt-out study that the detection of $\geqslant$ CIN2 in 1649 women who returned a self-sampling swab and in all screened women aged 30-69 years from the Australian state of Victoria were insignificantly different from each other. These findings could be explained by a shorter follow-up period in the self-sampling study (6 months) that might lower the number of $\geqslant$ CIN2 detected.

Our study had important strengths. Both the CSi and the Horizon studies were population based and included representative samples of routinely screened and routinely unscreened women. With the use of the national Patobank for identifying outcomes in both studies, our analysis had high data completeness in identifying the detected $\geqslant \mathrm{CIN} 2$ cases. This was the case even if the women moved outside of the laboratory's catchment area, thereby avoiding information bias. However, some limitations with regard to the comparability of the $\mathrm{CSi}$ and the Horizon studies need to be mentioned. Some of the CSi-attenders $(\mathrm{N}=41)$ and GPattenders $(\mathrm{N}=1029)$ in the CSi study had a follow-up time shorter than 1.5 years because they were screened very late; these women may have had $\geqslant$ CIN2 detected after the date of data retrieval. The two populations differed somewhat in terms of their respective screening catchment areas. The Horizon study covered the most urbanised parts of the Capital Region, whereas the CSi covered the whole Capital Region. Furthermore, the two studies varied in their design, HPV testing methods and triage procedures (Supplementary Table 1). Different HPV assays were used in the studies and the Horizon study showed that HPV detection depends on the assay (Rebolj et al, 2014b) that may affect the screening follow-up referrals (Rebolj et al, 2016a). For this analysis, all histological findings were retrieved, regardless of HPV results to ensure that all outcomes were captured. The CSi study was undertaken 3 years after the Horizon study. Changes in CIN incidence may occur over time; however, with only a few years 
Table 2. Odds ratios and $95 \%$ confidence intervals for $\geqslant \mathrm{CIN} 2$ detection, by age, study population, and screening history

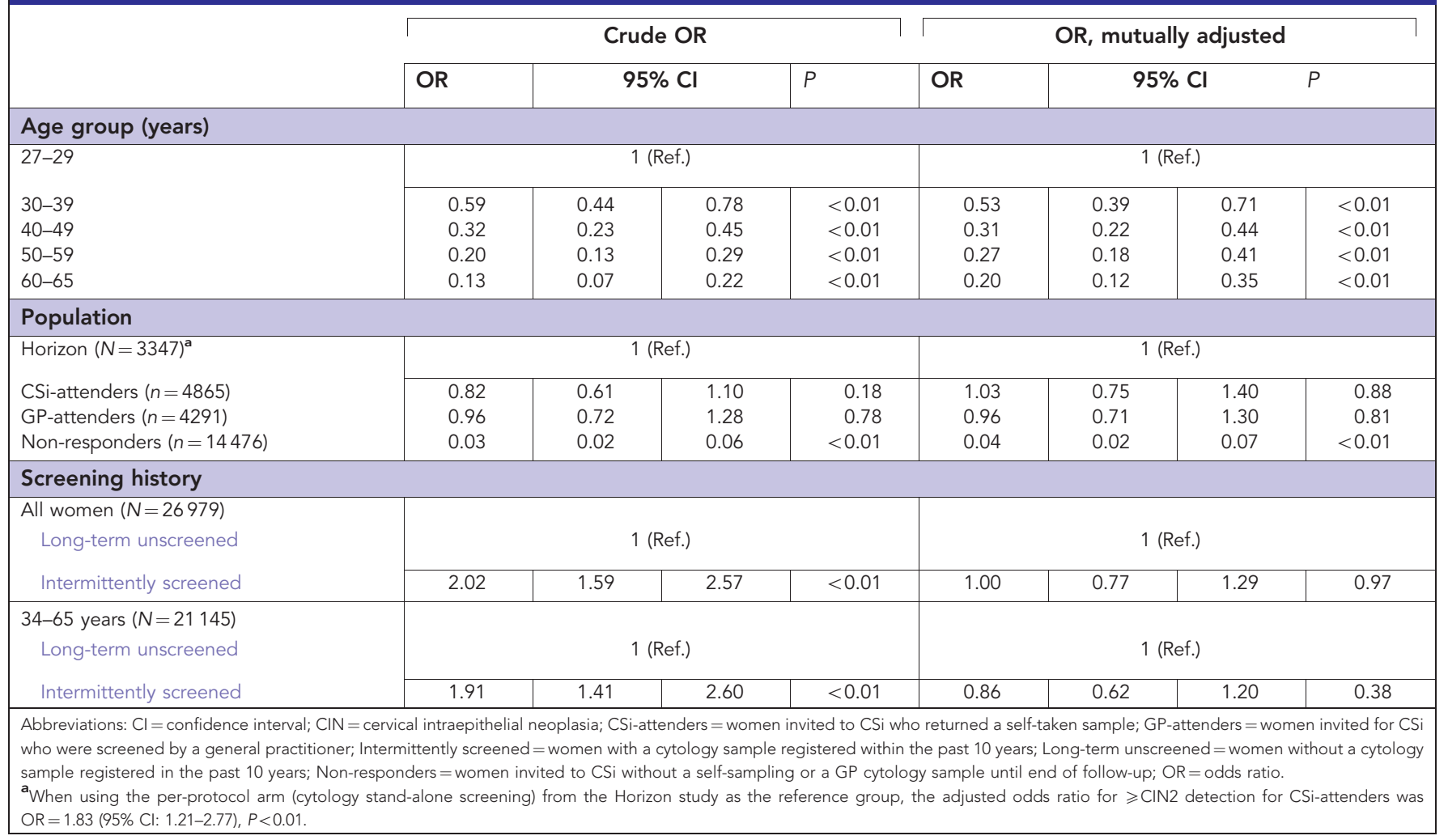

between the studies, we consider it unlikely. No changes in $\geqslant$ CIN2 detection over a 10-year period were previously found in the catchment area of the Roskilde laboratory that is in close geographical proximity to Copenhagen (Rebolj et al, 2015b). Primary screening recommendations also remained unchanged during those 3 years.

Based upon the resulting detection of $\geqslant \mathrm{CIN} 2$, self-sampling in this setting appears to be more effective than the current cytology routine screening, and would therefore lead to detection of an extra number of high-grade lesions requiring treatment. However, to finetune its implementation within an organised screening programme, several issues still need to be addressed. These include a definition of an optimal screening interval after a negative HPV self-sample that will be investigated by register-based monitoring of the screening and diagnostics activities of these women in the forthcoming years via the Patobank. Furthermore, we speculate that loss to follow-up could be reduced among self-sampling nonattenders if, for example, a direct referral to colposcopy is implemented for women with specific high-risk HPV genotypes replacing part of the current uniform referral for cytology followup. Finally, it is crucial for the integrity of an organised screening programme to foresee the proportions of women who may switch from GP-screening to self-sampling by skipping calls for screening in order to obtain a non-responder status (Rozemeijer et al, 2015). As organised cervical cancer screening depends on participation within regular intervals, the latter could over time develop into a challenge.

\section{CONCLUSION}

We observed higher detection rates of $\geqslant \mathrm{CIN} 2$ with HPV-based self-sampling than in the routine cytology-based screening, suggesting that self-sampling is a good screening alternative for non-attenders. Furthermore, the proportion of high-grade CIN lesions among all biopsies was high, suggesting that the chosen referral approach was efficient.

\section{ACKNOWLEDGEMENTS}

We thank pathologist Maria Benedicte Franzmann, MD, Chief Physician, for the invaluable help in validating histological diagnoses from the Patobank. Horizon was funded by Danish Strategic Research Council, Grant No. 10-092793; CSi was mandated and funded by the Capital Region of Denmark, and received public funding. BD Diagnostics, Sparks Circle, MD, USA, delivered reagents and instrumentation free of charge for the Onclarity HPV analysis. BD Diagnostics had no role in this work.

\section{CONFLICT OF INTEREST}

JUHL attended meetings with various HPV device manufacturers. MR attended meetings with various HPV device manufacturers. She and her former employer received fees for lectures on her behalf from Qiagen. DME attended meetings with various HPV device manufacturers. She received honoraria from BD and Qiagen for lectures. HP attended meetings with various HPV device manufacturers. CR participated in meetings with Roche with fee paid to the University of Copenhagen. EL participated in meetings with Roche and Astra-Zeneca with fees paid to the University of Copenhagen, and was unpaid advisor to GenProbe and NorChip. Roche has provided test kits to Trial23. SKK has received lecture fees, scientific advisory board fees from Merck, Sanofi Pasteur MSD, BD, and unrestricted research grants through the affiliating institute from Merck. JD has received grants to his institution for studies on HPV vaccination from Merck/SPMSD. JB attended 
meetings with various HPV device manufacturers. He used to serve as a paid advisor to Roche and Genomica, and has received honoraria from Hologic/Gen-Probe, Roche, Qiagen, Genomica, and $\mathrm{BD}$ Diagnostics for lectures. He is principal investigator on studies funded by BD diagnostics. Hvidovre Hospital holds a recompense agreement with Genomica on a KRAS/BRAF diagnostic system. KME and KEJ declare no conflict of interest.

\section{AUTHOR CONTRIBUTIONS}

Design of the study: JUHL, KME, MR, JD, and JB; analysis of the data: JUHL, KME, and JD; interpretation of the results, drafting of the manuscript, and decision to submit: all authors.

\section{REFERENCES}

Bais AG, van Kemenade FJ, Berkhof J, Verheijen RH, Snijders PJ, Voorhorst F, Babovic M, van Ballegooijen M, Helmerhorst TJ, Meijer CJ (2007) Human papillomavirus testing on self-sampled cervicovaginal brushes: an effective alternative to protect nonresponders in cervical screening programs. Int $J$ Cancer 120(7): 1505-1510.

Bjerregaard B, Larsen OB (2011) The Danish Pathology Register. Scand J Public Health 39(7 Suppl): 72-74.

Bonde J, Rebolj M, Ejegod DM, Preisler S, Lynge E, Rygaard C (2014) HPV prevalence and genotype distribution in a population-based split-sample study of well-screened women using CLART HPV2 human papillomavirus genotype microarray system. BMC Infect Dis 14: 413.

(2014) Danish Quality Assurance Database for the Cervical Cancer Screening Program Annual report 2014 Vol. 2015.

Dugue PA, Lynge E, Bjerregaard B, Rebolj M (2012) Non-participation in screening: the case of cervical cancer in Denmark. Prev Med 54(3-4): 266-269.

Ejegod DM, Rebolj M, Bonde J (2015) Comparison of analytical and clinical performance of CLART HPV2 genotyping assay to Linear Array and Hybrid Capture 2: a split-sample study. BMC Cancer 15: 216 .

Enerly E, Bonde J, Schee K, Pedersen H, Lonnberg S, Nygard M (2016) Selfsampling for human Papillomavirus testing among non-attenders increases attendance to the Norwegian cervical cancer screening programme. PLoS One 11(4): e0151978.

Gok M, Heideman DA, van Kemenade FJ, Berkhof J, Rozendaal L, Spruyt JW, Voorhorst F, Belien JA, Babovic M, Snijders PJ, Meijer CJ (2010) HPV testing on self collected cervicovaginal lavage specimens as screening method for women who do not attend cervical screening: cohort study. BMJ 340: c1040.

Gok M, Heideman DA, van Kemenade FJ, de Vries AL, Berkhof J, Rozendaal L, Belien JA, Overbeek L, Babovic M, Snijders PJ, Meijer CJ (2012) Offering self-sampling for human papillomavirus testing to non-attendees of the cervical screening programme: characteristics of the responders. Eur J Cancer 48(12): 1799-1808.
Lam JU, Rebolj M, Ejegod DM, Pedersen H, Rygaard C, Lynge E, Thomsen LT, Kjaer SK, Bonde J (2017) Prevalence of human papillomavirus in self-taken samples from screening nanottenders. J Clin Microbiol 55(10): 2913-2923.

Preisler S, Rebolj M, Ejegod DM, Lynge E, Rygaard C, Bonde J (2016) Crossreactivity profiles of hybrid capture II, cobas, and APTIMA human papillomavirus assays: split-sample study. BMC Cancer 16: 510 .

Preisler S, Rebolj M, Untermann A, Ejegod DM, Lynge E, Rygaard C, Bonde J (2013) Prevalence of human papillomavirus in 5,072 consecutive cervical SurePath samples evaluated with the Roche cobas HPV real-time PCR assay. PLoS One 8(3): e59765.

Rebolj M, Bonde J, Ejegod D, Preisler S, Rygaard C, Lynge E (2015a) A daunting challenge: human papillomavirus assays and cytology in primary cervical screening of women below age 30years. Eur J Cancer 51(11): 1456-1466.

Rebolj M, Bonde J, Preisler S, Ejegod D, Rygaard C, Lynge E (2016a) Differential detection of human papillomavirus genotypes and cervical intraepithelial neoplasia by four commercial assays. J Clin Microbiol 54(11): 2669-2675.

Rebolj M, Bonde J, Preisler S, Ejegod D, Rygaard C, Lynge E (2016b) Human papillomavirus assays and cytology in primary cervical screening of women aged 30 years and above. PLoS One 11(1): e0147326.

Rebolj M, Lynge E, Ejegod D, Preisler S, Rygaard C, Bonde J (2014a) Comparison of three human papillomavirus DNA assays and one mRNA assay in women with abnormal cytology. Gynecol Oncol 135(3): 474-480.

Rebolj M, Preisler S, Ejegod DM, Bonde J, Rygaard C, Lynge E (2013) Prevalence of human papillomavirus infection in unselected SurePath samples using the APTIMA HPV mRNA assay. J Mol Diag 15(5): 670-677.

Rebolj M, Preisler S, Ejegod DM, Rygaard C, Lynge E, Bonde J (2014b) Disagreement between human papillomavirus assays: an unexpected challenge for the choice of an assay in primary cervical screening. PLoS One 9(1): e86835.

Rebolj M, Rask J, van Ballegooijen M, Kirschner B, Rozemeijer K, Bonde J, Rygaard C, Lynge E (2015b) Cervical histology after routine ThinPrep or SurePath liquid-based cytology and computer-assisted reading in Denmark. Br J Cancer 113(9): 1259-1274.

Rozemeijer K, de Kok IM, Naber SK, van Kemenade FJ, Penning C, van Rosmalen J, van Ballegooijen M (2015) Offering self-sampling to nonattendees of organized primary HPV screening: when do harms outweigh the benefits? Cancer Epidemiol Biomarkers Prev 24(5): 773-782.

Sultana F, English DR, Simpson JA, Drennan KT, Mullins R, Brotherton JM, Wrede CD, Heley S, Saville M, Gertig DM (2016) Home-based HPV selfsampling improves participation by never-screened and under-screened women: Results from a large randomized trial (iPap) in Australia. Int $J$ Cancer 139(2): 281-290.

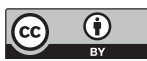

This work is licensed under the Creative Commons Attribution 4.0 International License. To view a copy of this license, visit http://creativecommons.org/licenses/by/4.0/

(C) The Author(s) named above 2018

Supplementary Information accompanies this paper on British Journal of Cancer website (http://www.nature.com/bjc) 